\title{
PROSPECTS FOR A 4TH GENERATION LIGHT SOURCE FOR THE UK
}

\author{
J. A. Clarke, H. L. Owen, M. W. Poole, S. L. Smith, V. P. Suller \& N. G. Wyles, \\ CLRC Daresbury Laboratory, Warrington, UK.
}

\begin{abstract}
A 4th generation light source (4GLS) forms a major part of the new CASIM (Centre for Accelerator Science, Imaging and Medicine) project that has been proposed to be based at Daresbury Laboratory. Such a light source is envisaged to contain 3 Free Electron Lasers (FELs) that are integrated with a highly optimised low energy synchrotron radiation source. The light source will be optimised to cover the photon energy range of 5 to $100 \mathrm{eV}$ as well as being designed to host a cavity based FEL that would operate in the UV region. A second, linac based, infrared FEL would be placed in the same building thus enabling pump-probe experiments to be carried out with state of the art photon flux and brightness. The third FEL would not initially be a user facility but would be a linac based SASE FEL research project with the aim of producing ultra high brightness light in the VUV/SXR region. The primary source of 5 to $100 \mathrm{eV}$ light could either be a storage ring or an energy recovery linac. Both options are presently being studied and a choice will be made by the Autumn of 2001. This paper describes the present concept for the 4GLS project and details the current status of the designs.
\end{abstract}

\section{BACKGROUND}

Following the decision in March 2000 that the new UK $3 \mathrm{GeV}$ synchrotron light source, DIAMOND [1], was to be built at Rutherford Appleton Laboratory the UK government announced that a review of science in the North West of England would be undertaken. Two review committees subsequently recommended several projects, which have since been funded, but the main recommendation was to establish a Centre for Accelerator Science, Imaging and Medicine (CASIM) at Daresbury Laboratory [2]. Such a centre would bring together many of the strengths of the UK science and engineering base and would link the existing infrastructure and scientific expertise at Daresbury Laboratory with hospitals, universities and industry.

A UK government statement in March 2001 announced support for the CASIM project at Daresbury Laboratory with funding being subject to the normal review process and the completion of the feasibility studies. At the heart of the CASIM project are two major new state of the art facilities for the UK. The first is a $230 \mathrm{MeV}$ proton cyclotron which will be used as a driver for a radioactive ion beam facility and a proton therapy centre. The second is a suite of accelerator based light sources of unparalleled intensity from the far-infrared to the extreme ultraviolet. As these light sources are largely Free Electron Laser (FEL) based this facility has been termed the 4th
Generation Light Source (4GLS). The layout and accelerator driver technology of the 4GLS facility has not yet been finalised and is the subject of this paper.

\section{THE 4GLS}

The 4GLS requirement is to provide state of the art photon output from the far infrared to the extreme ultraviolet. Such a requirement will be met by a suite of accelerator based light sources. The infrared region will be predominantly met by a stand-alone linac based infrared FEL. However, for the photon range of a few eV to a few hundred $\mathrm{eV}$ there are two basic options that are being considered presently. The first is a low energy storage ring $(900 \mathrm{MeV})$ that will provide diffraction limited undulator output and will also support a cavity based UVFEL operating in the range $\sim 3$ to $\sim 20 \mathrm{eV}$. The second is an Energy Recovery Linac based scheme that will again provide diffraction limited spontaneous radiation from undulators but will also provide continuously tuneable UV radiation from a SASE FEL. The possible layout of these two schemes will be examined in more detail below.

\subsection{Storage Ring Based Facility}

The basic specification for the storage ring is to have an energy of $900 \mathrm{MeV}$, an emittance of less than $10 \mathrm{nmrad}$ and at least 6 straight sections available for insertion devices with some of these being $\sim 10 \mathrm{~m}$ long.

The basic layout of a facility based upon a storage ring is independent of the exact lattice chosen. It is considered essential to have a full energy injector as the lifetime of the stored beam is unlikely to exceed $\sim 10$ hours and it also opens up the possibility of continuous top-up injection. Several schemes have been considered but the one with the greatest future upgrade potential whilst still being economically viable has a linac pre-injector coupled to a booster synchrotron. The upgradability comes from the careful choice of linac structure and layout to ensure that it is suitable for SASE FEL experiments. The energy of the linac could be increased later by adding more RF structures. A possible layout is shown in figure 1 . 


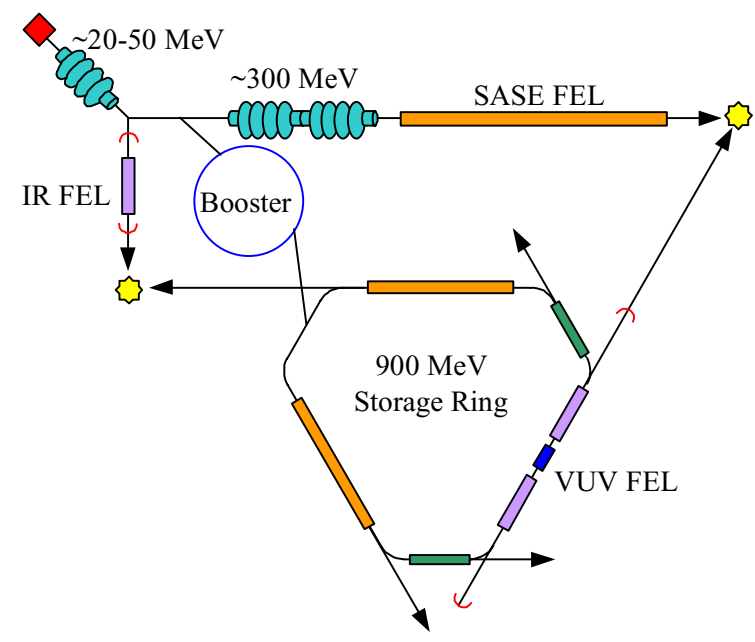

Figure 1. Schematic layout for storage ring based 4GLS. Possible combined pump-probe experiments have been highlighted.

Two possible lattice arrangements have been considered so far. The first is a conventional three-fold symmetric triple bend achromat (TBA) solution and the second is a lattice of combined function arcs with short matching sections to create dispersion free straights based upon the ASTRID II concept [3].

\subsubsection{TBA Lattice}

To reach the emittance requirement of less than $10 \mathrm{nmrad}$ with a TBA at least 6 achromat arcs are required. It was decided that a useful feature would be to have some very long straights, not least for the UVFEL, and so three $14 \mathrm{~m}$ long straights and three shorter $4 \mathrm{~m}$ straights were incorporated into a TBA lattice creating a basic 3 fold symmetric lattice. The circumference of this lattice is $\sim 150 \mathrm{~m}$.

\subsubsection{FODO Arc Lattice}

The ASTRID II lattice claimed to have superior dynamic behaviour over standard achromat lattices because of the careful phase advance per dipole cell chosen to give cancellation of non-linear terms. With all the sextupoles in the arc sections the dispersion free straight sections are effectively decoupled from the bends creating a more flexible lattice solution. Our lattice has four arcs and phase advances of $90^{\circ}$ per dipole cell were set both radially and vertically. These arcs were matched into four $14 \mathrm{~m}$ straight sections though it is envisaged that some of these could be split into two shorter straights $(2 \mathrm{x} \sim 5 \mathrm{~m})$ by using a short achromat section at the centre of the straight. The parameters of the two lattice types under consideration are given in Table 1. The FODO Arc lattice has a much smaller circumference of $116 \mathrm{~m}$ but the restriction on the fixed phase advance per dipole cell appears to limit the number of working points.
Table 1. Possible Storage Ring parameters.

\begin{tabular}{|c|c|c|}
\hline & TBA Lattice & $\begin{array}{c}\text { FODO Arc } \\
\text { Lattice }\end{array}$ \\
\hline Energy $(\mathrm{GeV})$ & 0.9 & 0.9 \\
\hline Circumference $(\mathrm{m})$ & 147.9 & 116.0 \\
\hline Number of Cells & 3 & 4 \\
\hline $\begin{array}{c}\text { Straight Section } \\
\text { Length }(\mathrm{m})\end{array}$ & $\begin{array}{c}3 \times 14 \\
\text { and } 3 \times 4\end{array}$ & $\begin{array}{c}4 \times 2 \times 5 \\
\text { or } 4 \times 14\end{array}$ \\
\hline Emittance $(\mathrm{nmrad})$ & 6.1 & 3.2 \\
\hline Beam Current $(\mathrm{mA})$ & 300 & 300 \\
\hline Bunch Length $(\mathrm{ps})$ & $20-50$ & $20-50$ \\
\hline Bunch Spacing $(\mathrm{ns})$ & 2 & 2 \\
\hline
\end{tabular}

\subsection{Energy Recovery Linac Based Facility}

An alternative to the storage ring scheme is to accelerate electrons through a medium energy linac (few hundred $\mathrm{MeV}$ ) and then pass them along a transfer path containing spontaneous emission undulators or a SASE FEL. To make such a scheme viable the electrons would not be dumped at high energy but passed once more through the linac at a deceleration phase such that their energy is transferred to the RF structure and is available for a fresh set of electrons. This concept for a synchrotron light source formed the basis of the MARS proposal [4] and is now being considered by other groups [5]. A schematic layout is shown in figure 2 .

The advantages of the energy recovery linac (ERL) scheme are that the beam emittance decreases linearly with the electron energy, most instabilities can be neglected because there are no multiple passes to contend with, smaller ID gaps can be tolerated because the ERL effectively has continuous top-up and the electron bunch lengths can be sub-picosecond. It would also be possible to pass the electron beam through the accelerating structure a second time in phase, thus increasing its energy to a level suited to a different synchrotron radiation emission spectrum.

Since the emittance of the ERL is effectively determined by the emittance of the injected electron beam the electron source is a vital item for determining the final beam quality. It would seem that to provide the smallest possible injected beam emittance a laser photocathode RF gun must be used. A normalised emittance of $\sim 1 \mathrm{~mm}$ mrad with a high output current is now a realistic target.

Table 2 summarises the tentative ERL parameters that are presently being considered. The change from $900 \mathrm{MeV}$ for the storage ring to $500 \mathrm{MeV}$ for the ERL reflects the fact that the ERL can have much smaller gaps in the insertion devices and so both sources actually cover the same photon energy range. 
Table 2. Possible Energy Recovery Linac parameters.

\begin{tabular}{|c|c|}
\hline Beam Energy (MeV) & 500 \\
\hline Circumference (m) & $\sim 100$ \\
\hline Straight Section Length (m) & 5 to 25 \\
\hline Emittance at 500 MeV (nm rad) & $\sim 1$ \\
\hline Average Beam Current (mA) & 100 \\
\hline Peak Current (A) & $\sim 100$ \\
\hline Bunch Length (ps) & $<1$ \\
\hline Bunch Spacing (ns) & 0.77 \\
\hline
\end{tabular}

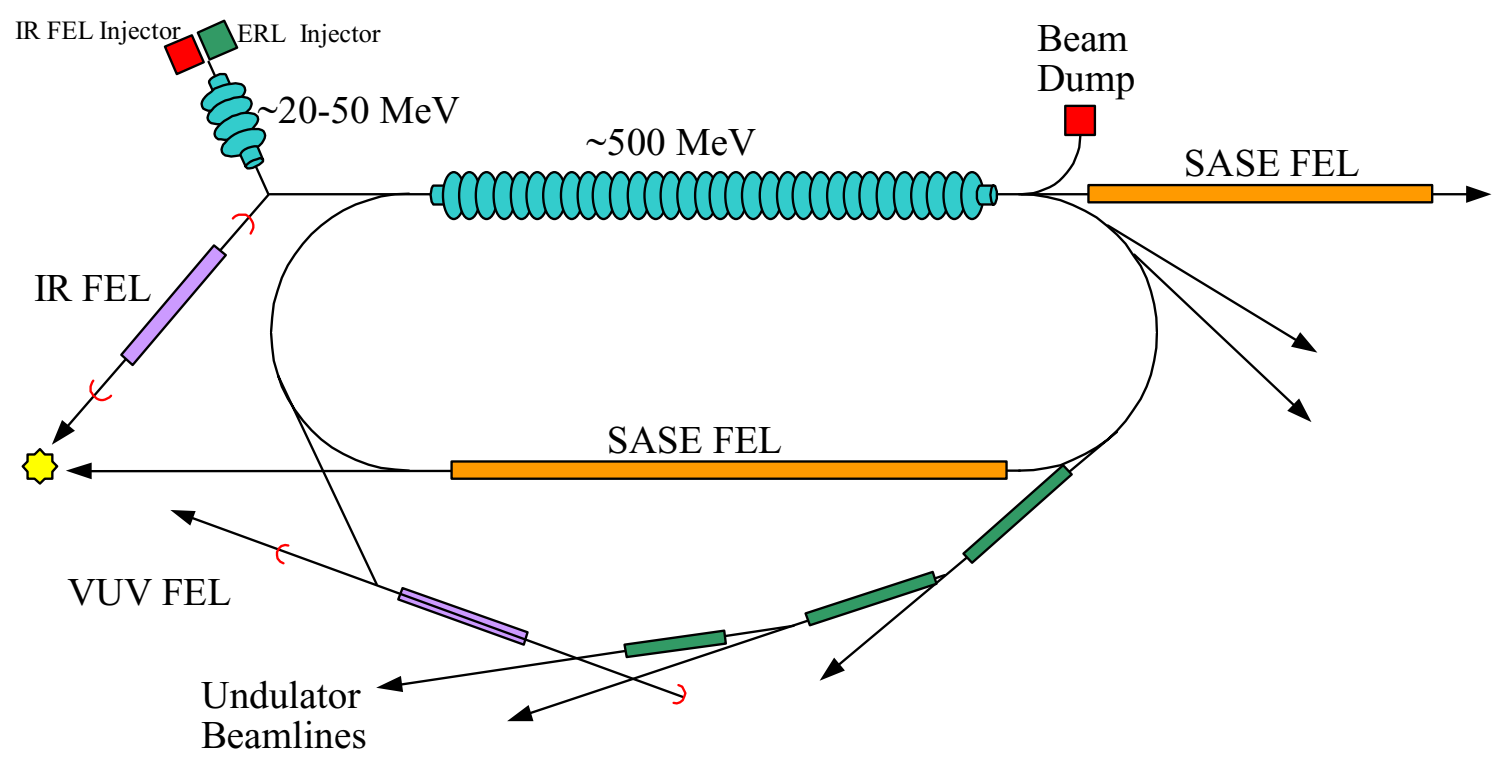

Figure 2. Schematic layout for energy recovery linac based 4GLS.

\section{FURTHER WORK}

Before a decision can be made between the two options under consideration for the 4GLS much further work is required. Each option needs to be studied in more detail to check how they each match the requirements of the UK science base.

Both the storage ring and ERL options appear to be feasible at the present time. Clearly the storage ring option contains fewer unknowns as there are many successful examples of highly optimised electron storage rings in operation around the world. The high current, low emittance ERL has yet to be demonstrated at a high energy though a low energy version has operated successfully [6] and the technology required appears to be available today. The ERL would also seem to have the greater potential for future upgrades and expansion.

Further studies will continue over the next few months before the full 4GLS and CASIM project proposal is formally submitted for approval to the British Government.

\section{REFERENCES}

[1] M. W. Poole et al, "The DIAMOND Project: An Advanced Light Source for the UK", these proceedings.

[2] http://www.casim.ac.uk/

[3] Y. Senichev, "The Proposed Racetrack Lattice for the Synchrotron Light Source Astrid II", EPAC 98, Stockholm, June 1998, p641.

[4] D. A. Kayran et al, "MARS - A Project of the Diffraction Limited Fourth Generation X-Ray Source", APAC 98, Tsukuba, March 1998, p704.

[5] I. Ben-Zvi \& S. Krinsky, "Future Light Sources based upon Photo-injected Energy Recovery Linacs", SRN, Vol. 14, No. 2, p20 (2001).

[6] G. R. Neil et al, "Sustained Kilowatt Lasing in a FreeElectron Laser with Same-Cell Recovery", Phys. Rev. Lett., 84, 4 (2000), p662. 